\title{
Marcha en inversión
}

\section{Intoeing gait}

Alfonso Migoya-Nuño, ${ }^{1}$ José Pablo Unda-Haro, ${ }^{2}$ Pola Capuano-Tripp ${ }^{3}$

El caminar de los niños, conocido como marcha, no es homogéneo,

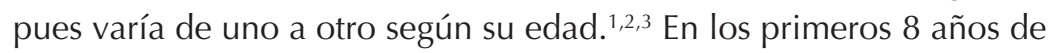
vida la marcha va perfeccionándose a medida que se adquieren habilidades, hasta que logran caminar de forma similar a los adultos. ${ }^{4,5}$

En este lapso, una variante muy frecuente es la "marcha en inversión de pies", que se define como el desplazamiento o rotación de las puntas de los pies hacia la línea media. 6,2,7,8 Figura $\mathbf{1}$

La marcha en inversión de pies suele originarse por alguna de las siguientes causas: 1) aducto de antepié, 2) torsión tibial o 3) anteversión femoral ${ }^{4,9,10,11}$ y cada una puede preponderar en un determinado límite de edad. ${ }^{8,6,5,12}$ Cuadro 1

La marcha en inversión de pies representa una de las principales causas de envío al ortopedista pediátrico, ${ }^{3,2}$ por lo que vale la pena hacer una revisión que permita al pediatra conocer las diferentes causas y su historia natural, para diferenciar los casos que simplemente requieren vigilancia de los que ameritan alguna intervención.

Existe una ligera predisposición en mujeres e hiperlaxitud ligamentaria, ${ }^{8}$ casi siempre aparece de manera bilateral, con predominio izquierdo, ${ }^{13}$ que suele tener en promedio $6 \pm 3$ grados más de rotación interna que el derecho. ${ }^{14}$

\section{Aducto de antepié}

Deformidad congénita causada por aducción del antepié, en la articulación metatarsal, donde el retropié tiene adecuada alineación. ${ }^{5,15,8} \mathrm{Su}$ origen se relaciona con la posición intrauterina; ${ }^{6}$ los factores de riesgo incluyen: madre primigesta, partos gemelares y oligohidramnios. ${ }^{16} \mathrm{Se}$ manifiesta en 1 de cada 1000 recién nacidos vivos, ${ }^{17}$ sin predilección

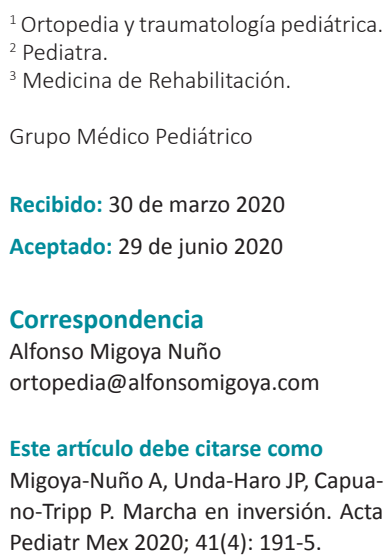


por el género y es bilateral en $50 \%$ de los casos; el diagnóstico es clínico, por lo que no están indicadas las radiografías. ${ }^{18,8}$ Cuadro 2

De acuerdo con el grado de afectación se clasifica en leve, moderado y severo. ${ }^{13,18}$ Figura 2 Sin importar su severidad, $90 \%$ de los casos se resuelve sin tratamiento, en los primeros 4 años de vida, y $5 \%$ se recupera a los 6 años de edad. ${ }^{2}$ El tratamiento está indicado sólo cuando la deformidad es rígida y ocasiona dolor, ${ }^{6,18}$ y consiste en enyesado seriado por el ortopedista pediatra.

\section{Torsión tibial interna}

Es la rotación interna de la tibia sobre su eje longitudinal. Se genera por la desviación del pie hacia la línea media. ${ }^{3,19,20}$

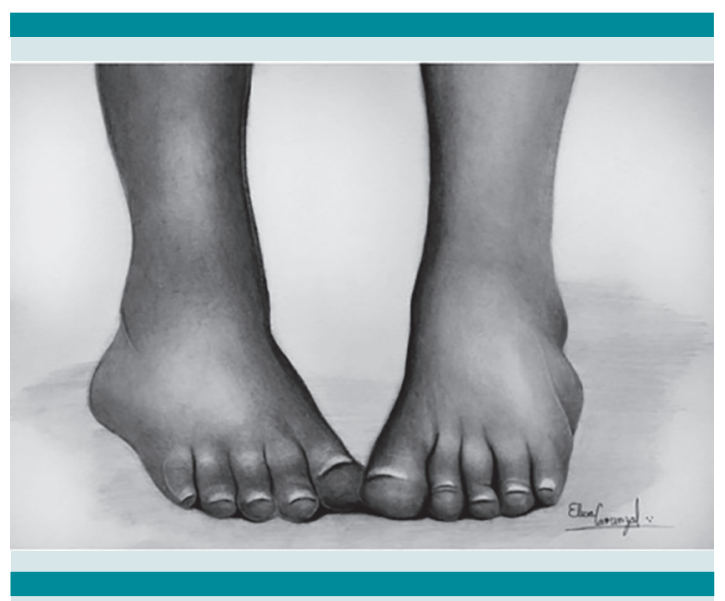

Figura 1. Posición típica que adoptan los pies durante la marcha en inversión, independientemente de su causa (aducto de antepié, torsión tibial o anteversión femoral).

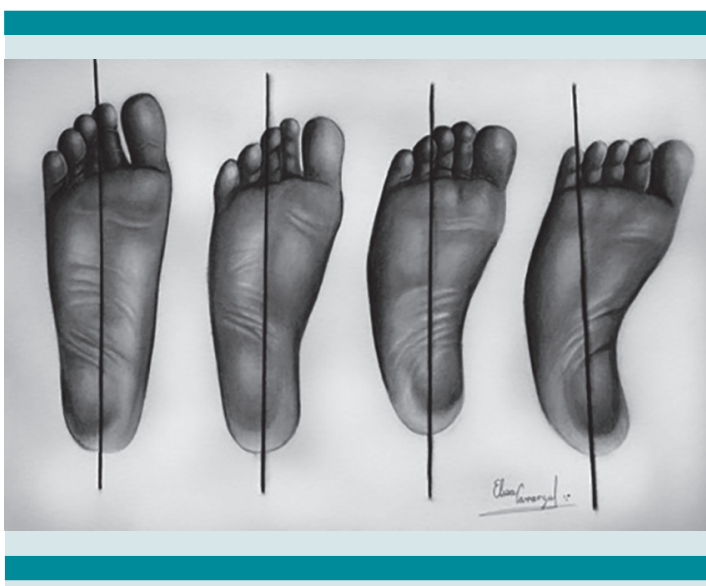

Figura 2. Línea transcalcánea en un pie normal y en casos de pie aducto leve, moderado y severo; de manera normal esta línea debe pasar sobre el segundo espacio interdigital; con la aducción del pie la línea se desplaza en sentido lateral hacia el quinto dedo.

Es la causa más común de marcha en inversión de pies entre el primer y cuarto años de vida, ${ }^{8,7,3}$ su etiología es desconocida ${ }^{2,18}$ y se considera una variante fisiología ${ }^{12}$ cuando aparece en este grupo de edad. Ocurre de forma bilateral en $75 \%$ de los casos, y la extremidad izquierda suele ser la más afectada en severidad y frecuencia; $;^{13}$ no guarda predisposición por algún género ni se asocia con dolor, pero sí con caídas frecuentes. ${ }^{21,12}$ El 30\% de los casos se relaciona con metatarso aducto y $90 \%$ se resuelve de manera espontánea, dentro de los primeros 5 años de vida. ${ }^{6}$

El diagnóstico es clínico ${ }^{6,8}$ y se establece al observar la desviación de los pies hacia la línea media en la posición de pie y durante la marcha, mien-

Cuadro 1. Características clínicas de la marcha en inversión

\begin{tabular}{|l|c|c|c|}
\hline Edad de manifestación & Segmento afectado & Etiología & Corrección espontánea \\
\hline 0-1 años & Pie & Aducto de antepié & 18 meses \\
\hline 1-3 años & Tibia & Torsión tibial & 4 años \\
\hline 3-6 años & Cadera & Anteversión femoral & 8 años \\
\hline
\end{tabular}




\begin{tabular}{|c|c|c|c|}
\hline Edad de manifestación & Segmento afectado & Etiología & Corrección espontánea \\
\hline 0-1 años & Pie & Idiopática-Postural & 18 meses \\
\hline \multicolumn{4}{|c|}{$\begin{array}{l}\text { Exploración física } \\
\text { Desviación del antepié en sentido medial, mientras que el } \\
\text { retropié se encuentra en neutro. }\end{array}$} \\
\hline \multicolumn{4}{|l|}{$\begin{array}{l}\text { Pruebas diagnósticas } \\
\text { Línea transcalcánea, sig } \\
\text { en el centro del calcáne }\end{array}$} \\
\hline
\end{tabular}

Envío a ortopedia: Deformidad rígida asociada con dolor y aducto severo que persiste, incluso, a los 2 años; deformidad persistente o progresión de la misma.

tras que la rótula respeta su alineación neutra. Al observar al paciente de pie, se visualiza que las puntas de los pies están desviadas hacia la línea media, mientras que las rodillas se mantienen en posición neutra, lo que es valorable al observar la posición de la rótula. ${ }^{9}$ Cuadro 3

En la posición decúbito prono es posible realizar la medición del ángulo muslo-pie (Figura 3), cuyo rango normal es de $5^{\circ}$ al nacimiento con progresión hasta $15^{\circ}$, ángulos mayores de $20^{\circ}$ deben considerarse patológicos y requieren la valoración del ortopedista pediatra. ${ }^{9,6}$

\section{Anteversión femoral}

La anteversión femoral es la rotación entre el cuello y la diáfisis femoral; al nacimiento, el ángulo es de $40^{\circ}$ y disminuye a $30^{\circ}$ en los primeros 2 años de vida, para posteriormente disminuir $1.5^{\circ}$ cada año hasta los 10 años, hasta alcanzar los $15^{\circ}$ (valor que será normal para la edad adulta). ${ }^{10,2,20}$

Entre los 4 y 7 años de edad representa la causa más común de marcha en inversión; se manifiesta cuando no se alcanza la disminución normal de este ángulo o cuando empeora debido a vicios posturales, en concreto la posición

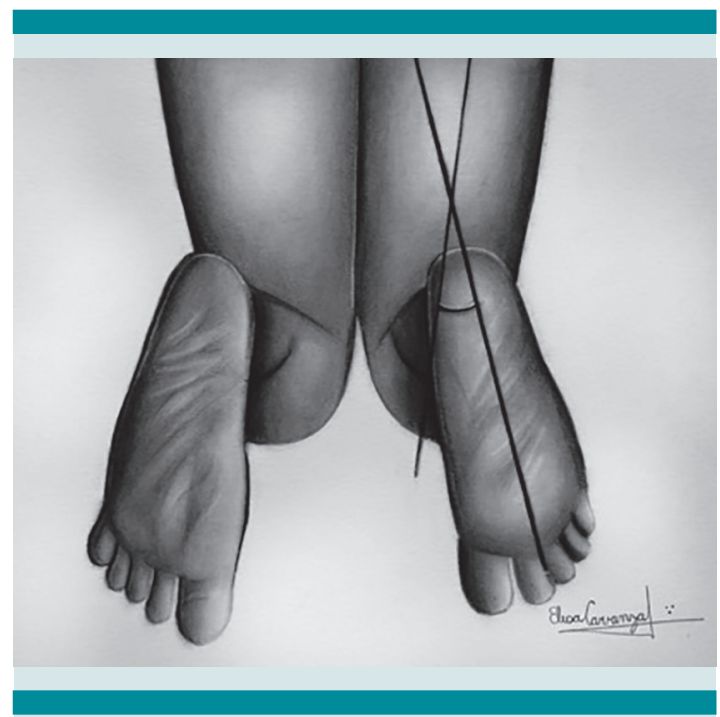

Figura 3. Ángulo del muslo-pie, que se genera de la intersección de las líneas trazadas sobre el eje longitudinal del pie y del muslo, cuando el paciente se encuentra en decúbito prono, con flexión de la rodilla a 90 grados y pies relajados o en posición neutra. La alteración de esté ángulo se presenta en torsión tibial.

de sedestación en $\mathrm{W}$ y la posición decúbito supino. ${ }^{5,20,2}$ Cuadro 3 El diagnóstico es clínico, al medir la anteversión femoral con el paciente en posición decúbito prono, considerando anormal los ángulos mayores a $40^{\circ}$ (Figura 4). La mayoría de los pacientes se repone de forma espontánea, con el simple hecho de evitar los vicios posturales; sin embargo, los pacientes 
Cuadro 3. Torsión tibial

\section{Edad de manifestación}

Segmento afectado

Tibia
Etiología

Idiopático

\section{Corrección espontánea}

1-3 años

Con el paciente de pie, se observa desviación del antepié en sentido medial, mientras que la rótula mantiene la alineación neutra.

\section{Pruebas diagnósticas}

Angulo del muslo-pie, con el paciente en decúbito prono, se mide el ángulo resultante de las líneas longitudinales que atraviesan en el muslo y el pie, cuyo valor normal es de $-5^{\circ}$ para menores de 2 años y de $30^{\circ}$ para mayores de 5 años.

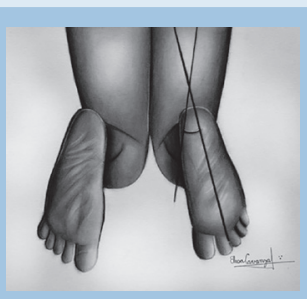

Envío a ortopedia: Mayores de 8 años con deformidad que genera trastorno funcional o cosmético, deformidad persistente o progresión de la misma.

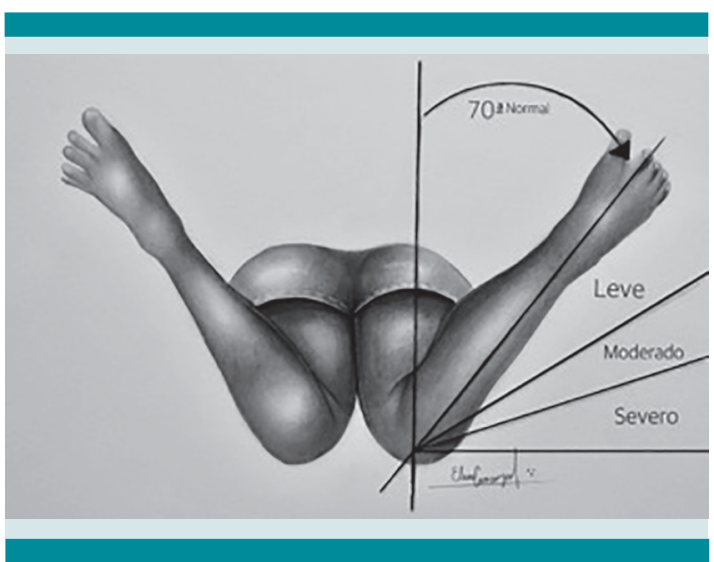

Figura 4. Prueba de anteversión femoral, con el paciente en decúbito prono y flexión de las rodillas entre 90 y $120^{\circ}$; de manera suave se rotan las piernas dejándolas caer en sentido lateral hasta sentir resistencia, en ese punto se mide el ángulo generado entre el eje longitudinal del muslo y de la pierna.

Cuadro 4. Anteversión femoral con anteversión mayor de 50 , edad mayor de 11 años y limitación funcional o estética requieren la valoración del ortopedista pediatra, por ser ideales a recibir tratamiento quirúrgico. 6,20 Como se comentó anteriormente, en todos los casos el diagnóstico es clínico y se resume en los Cuadros 2, 3 y 4.

El diagnóstico diferencial más común es la parálisis cerebral infantil, por lo que la evaluación del neurodesarrollo es decisiva en este tipo de pacientes; también debe descartarse: displasia del desarrollo de cadera, pie equino varo aducto y genu valgo.

El envío al ortopedista pediatra se resume en los Cuadros 2, 3 y 4.

Edad de manifestación
$\begin{aligned} & \text { 3-6 años } \\ & \text { Exploración física }\end{aligned}$
$\begin{aligned} & \text { Con el paciente de pie, se observa desviación del antepié en sen- } \\ & \text { tido medial; comparada con la torsión tibial las rótulas también se } \\ & \text { encuentran en rotación medial. }\end{aligned}$
Pruebas diagnósticas
Prueba de anteversión femoral, con el paciente en decúbito prono se
flexiona la rodilla $a 0^{\circ}$ y se deja caer haciosturales la mesa de exploración,
posteriormente se miden los grados de desplazamiento de la pierna.

Envío a ortopedia: paciente mayor de 8 años con deformidad que genera trastorno funcional o cosmético. Anteversión clínica mayor de $80^{\circ} \mathrm{o}$ anteversión radiográfica mayor de $50^{\circ}$, deformidad persistente o progresión de la misma. 


\section{REFERENCIAS}

1. Schulz JF, et al. Parental understanding of intoeing gait - A preliminary study. Foot 2019;41:39-43. https://doi. org/10.1016/j.foot.2019.06.004

2. Davis $L$, et al. Addressing pediatric intoeing in primary care. Nurse Pract. 2018;43(7):31-5. DOI: 10.1097/01. NPR.0000534939.42714.d0

3. Sielatycki JA, et al. In-Toeing Is Often a Primary Care Orthopedic Condition. J Pediatr 2016;177:297-301. http:// dx.doi.org/10.1016/j.jpeds.2016.06.022

4. Kahf $\mathrm{H}$, et al. Approach to pediatric rotational limb deformities. Orthop Rev (Pavia) 2019;11(3):99-101. DOI: 10.4081/or.2019.8118

5. Aston JW. In-toeing gait in children. Am Fam Physician. 1979;19(5):111-7.

6. Harris E. The Intoeing Child. Etiology, Prognosis, and Current Treatment Options. Clin Podiatr Med Surg. 2013;30(4):531-65. http://dx.doi.org/10.1016/j. cpm.2013.07.002

7. Boyer ER, et al. Gait pathology subtypes are not associated with self-reported fall frequency in children with cerebral palsy. Gait Posture 2018;63:189-94. https://doi. org/10.1016/j.gaitpost.2018.05.004

8. Rerucha $\mathrm{CM}$, et al. Lower extremity abnormalities in children. Am Fam Physician. 2017;96(4):226-33. https:// www.aafp.org/afp/2017/0815/p226.html

9. Blackmur JP, et al. Do children who in-toe need to be referred to an orthopaedic clinic? J Pediatr Orthop Part B. 2010;19(5):415-7. DOI: 10.1097/BPB.0b013e3283339067

10. Kong $M$, et al. Change of femoral anteversion angle in children with intoeing gait measured by three-dimensional computed tomography reconstruction: One-year follow-up study. Ann Rehabil Med. 2018;42(1):137-44. DOI: 10.5535/arm.2018.42.1.137
11. Kim HD, et al. Relationship between physical examinations and two-dimensional computed tomographic findings in children with intoeing gait. Ann Rehabil Med. 2011;35(4):491. DOI: 10.5535/arm.2011.35.4.491

12. Jones $\mathrm{S}$, et al. Normal variants of the lower limbs in pediatric orthopedics. Int J Clin Med. 2013;04(07):12-7. https://file. scirp.org/pdf/IJCM_2013071614061458.pdf

13. Lincoln TL, eSuen PW. Common rotational variations in children. J Am Acad Orthop Surg. 2003;11(5):312-20. DOI: 10.5435/00124635-200309000-00004

14. Verch $\mathrm{R}$, et al. Is in-toing gait physiological in children? - Results of a large cohort study in 5910 healthy (pre-) school children. Gait Posture. 2018;66(August):70-5. DOI: 10.1016/j. gaitpost.2018.08.019

15. Dawoodi AIS, et al. Radiological assessment of metatarsus adductus. Foot Ankle Surg. 2012;18(1):1-8. http://dx.doi. org/10.1016/j.fas.2011.03.002

16. Price MA, et al. Growth and development. Bioenerg Wild Herbiv. 2018;183-214. DOI: 10.2307/3899186

17. Eamsobhana $\mathrm{P}$, et al. Does the parental stretching programs improve metatarsus adductus in newborns? J Orthop Surg. 2017;25(1):1-5. https://journals.sagepub.com/doi/ full/10.1177/2309499017690320

18. Davids JR, et al. Surgical management of persistent intoeing gait due to increased internal tibial torsion in children. J Pediatr Orthop. 2014;34(4):467-73. DOI: 10.1097/ BPO.0000000000000173

19. Faulks S, et al. Spectrum of diagnosis and disposition of patients referred to a pediatric orthopaedic center for a diagnosis of intoeing. J Pediatr Orthop. 2017;37(7):e432-5. DOI: 10.1097/BPO.0000000000001007

20. Mooney JF. Lower Extremity Rotational and Angular Issues in Children. Pediatr Clin North Am. 2014;61(6):1175-83. http:// dx.doi.org/10.1016/j.pcl.2014.08.006

21. Mouri $\mathrm{H}$, et al. Effectiveness of medial-wedge insoles for children with intoeing gait who fall easily. Turkish J Phys Med Rehabil. 2019;65(1):9-15. 